\title{
Branched carbon nanofiber network synthesis at room temperature using radio frequency supported microwave plasmas
}

\author{
Bojan O. Boskovic, ${ }^{\text {a) }}$ Vlad Stolojan, Dagou A. Zeze, Roy D. Forrest, and S. Ravi P. Silva ${ }^{\text {b) }}$ \\ Advanced Technology Institute, School of Electronics and Physical Sciences, University of Surrey, \\ Guildford, Surrey, GU2 7XH, United Kingdom
}

Sajad Haq

Advanced Technology Centre, Sowerby Building, BAE SYSTEMS, FPC 267, PO Box 5, Filton, Bristol, BS34 7QW, United Kingdom

(Received 4 March 2004; accepted 2 June 2004)

\begin{abstract}
Carbon nanofibers have been grown at room temperature using a combination of radio frequency and microwave assisted plasma-enhanced chemical vapor deposition. The nanofibers were grown, using Ni powder catalyst, onto substrates kept at room temperature by using a purposely designed water-cooled sample holder. Branched carbon nanofiber growth was obtained without using a template resulting in interconnected carbon nanofiber network formation on substrates held at room temperature. This method would allow room-temperature direct synthesized nanofiber networks over relatively large areas, for a range of temperature sensitive substrates, such as organic materials, plastics, and other polymers of interest for nanoelectronic two-dimensional networks, nanoelectromechanical devices, nanoactuators, and composite materials. (c) 2004 American Institute of Physics. [DOI: 10.1063/1.1775300]
\end{abstract}

\section{INTRODUCTION}

The considerable scientific interest that has arisen in carbon nanostructures since buckminsterfullerene ${ }^{1}$ and carbon nanotubes $(\mathrm{CNFs})^{2}$ were identified has led to numerous investigations into their electronic and mechanical properties and possible engineering applications. Catalytic chemical vapor deposition of hydrocarbons is now widely used for carbon nanotube growth as a simple and efficient method for their production. In addition to CNTs, similar methods have been used for the synthesis of carbon nanofibers (CNFs), also known as carbon filaments since the early 1950's. ${ }^{3}$ CNFs can be grown using catalytic decomposition of hydrocarbons over transition metal particles such as iron, cobalt, nickel, and their alloys at temperatures ranging from 500 to $1000{ }^{\circ} \mathrm{C} .{ }^{4} \mathrm{~A}$ microwave plasma enhanced chemical vapor deposition (PECVD) process, used for the preparation of diamond and diamond-like carbon films, has recently been used for the growth of CNTs and CNFs. ${ }^{5-10}$ However, in all these methods reported substrate temperatures were higher than $500{ }^{\circ} \mathrm{C}$ due to the microwave plasma heating and use of additional heating source.

The formation of carbon nanotubes and nanofiber interconnecting networks with excellent electrical ${ }^{11}$ and thermal conduction ${ }^{12}$ could improve these parameters, even at low concentrations, when used as conductive filling material in composite structures. The synthesis of connections between two or more different CNFs is important for the development of carbon nanotube-based electronic devices and conducting structures. ${ }^{13-17}$ Branched carbon nanotubes have been synthesized using pyrolysis at temperatures of around

\footnotetext{
${ }^{\text {a)} P r e s e n t ~ a d d r e s s: ~ D e p a r t m e n t ~ o f ~ E n g i n e e r i n g, ~ U n i v e r s i t y ~ o f ~ C a m b r i d g e, ~}$ Trumpington Street, Cambridge, CB2 1PZ, United Kingdom.

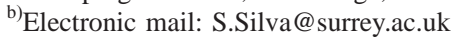

$1000{ }^{\circ} \mathrm{C} .{ }^{18,19}$ Multijunction carbon nanotube networks have been also synthesized using pyrolysis at $1100{ }^{\circ} \mathrm{C} .{ }^{20}$ Using an alumina template with branched channels produced by etching, ${ }^{14} Y$-shaped junctions of carbon nanotubes were synthesized by CVD at $650{ }^{\circ} \mathrm{C}$. For carbon nanotubes synthesized using radio frequency PECVD (rf PECVD) at around $650{ }^{\circ} \mathrm{C}$, nanojunction formation was observed after the application of high electric field. ${ }^{21}$

In order to enable use of polymers and organic substrates (for the CNT and CNF growth) that cannot tolerate higher process temperatures, we have developed a method for catalytic CNF growth at room temperature using radio frequency combined with microwave PECVD, where the substrate was kept at room temperature by using a purposely designed water-cooled sample holder. Instead of using high temperatures $\left(>500{ }^{\circ} \mathrm{C}\right)$ to provide the correct growth environment for the CNFs, we have utilized hydrocarbon plasmas to provide the energy dynamics necessary for the dissociation of the carbon and the subsequent catalytic growth on transition metal particles at room temperature.

Recently we published the evidence of carbon nanofiber growth at room temperature using rf PECVD. ${ }^{22}$ With an additional microwave power source, used in this work, significant numbers of branched or welded CNFs, forming interconnecting networks, was synthesized at room temperature. This method should allow the growth of CNTs and CNFs over relatively large areas on different substrates including plastics and other polymers which are extremely important for potential applications such as flat panel displays ${ }^{23}$ and fuel cells. ${ }^{24}$ Growth of aligned carbon nanofibers on plastic polyimide foil substrates for flat panel display applications has been reported recently. ${ }^{23}$ However, the growth temperature of $200^{\circ} \mathrm{C}$ is still restricting the use of some temperature sensitive plastic and organic substrates that room tempera- 


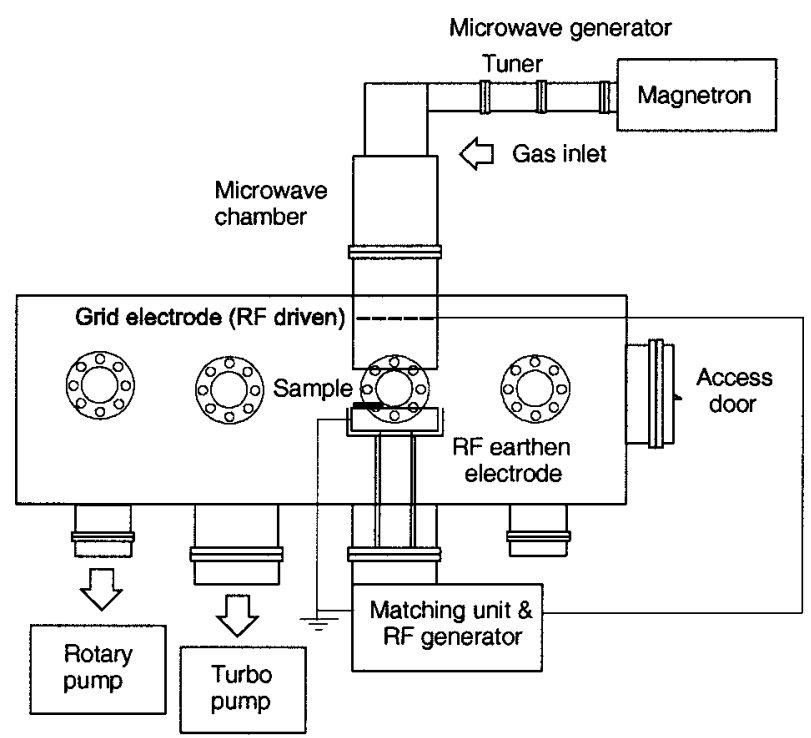

FIG. 1. A schematic of the radio frequency supported microwave PECVD system used to grow carbon nanofibers.

ture growth conditions will allow. The method we have used in this work uniquely demonstrates direct synthesis of branched and nonbranched carbon nanofibers and their interconnecting networks at room temperature.

\section{EXPERIMENTAL DETAILS}

The CNFs were grown using a $13.56 \mathrm{MHz}$ radio frequency PECVD system, supported by a $2.45 \mathrm{GHz}$ microwave power supply (Fig. 1), at $100 \mathrm{~W}$ rf power and 250-850 W microwave power. For the given process conditions, the microwave and radio frequency plasma input power was kept constant during the deposition. Nickel powder (99\% purity), with average particle diameter of 4-7 $\mu \mathrm{m}$, was used as a catalyst in the CNF growth process. Approximately $50 \mathrm{mg}$ of nickel powder was dispersed to produce a uniform thin layer on the top of a graphite or silicon substrate. In order to keep the macroscopic substrate temperature $\left(25^{\circ} \mathrm{C}\right)$ near constant, the substrate was placed on a water-cooled sample holder and the temperature was monitored. Methane gas (99.999\% purity) with a flow rate of $70 \mathrm{sccm}$ was introduced into the chamber prior to switching on the radio frequency and microwave power supplies. Experiments were conducted for $15 \mathrm{~min}$ at a process pressure of 12 mTorr.

The CNFs were characterized using a Hitachi S-4000 field emission scanning electron microscope (SEM) and a Philips CM 200 transmission electron microscope (TEM) equipped with a Gatan Imaging Filter energy loss spectrometer.

\section{RESULTS AND DISCUSSION}

Analysis conducted using SEM and TEM have shown that the average length of the CNFs is between 0.5 and $20 \mu \mathrm{m}$ with diameter ranging from 10 to $100 \mathrm{~nm}$ with average diameter around $50 \mathrm{~nm}$ [Fig. 2(a)]. Nickel catalyst particles were observed at the end of the CNFs, remote from the substrate (Fig. 2). The diameter of the CNF is similar to that
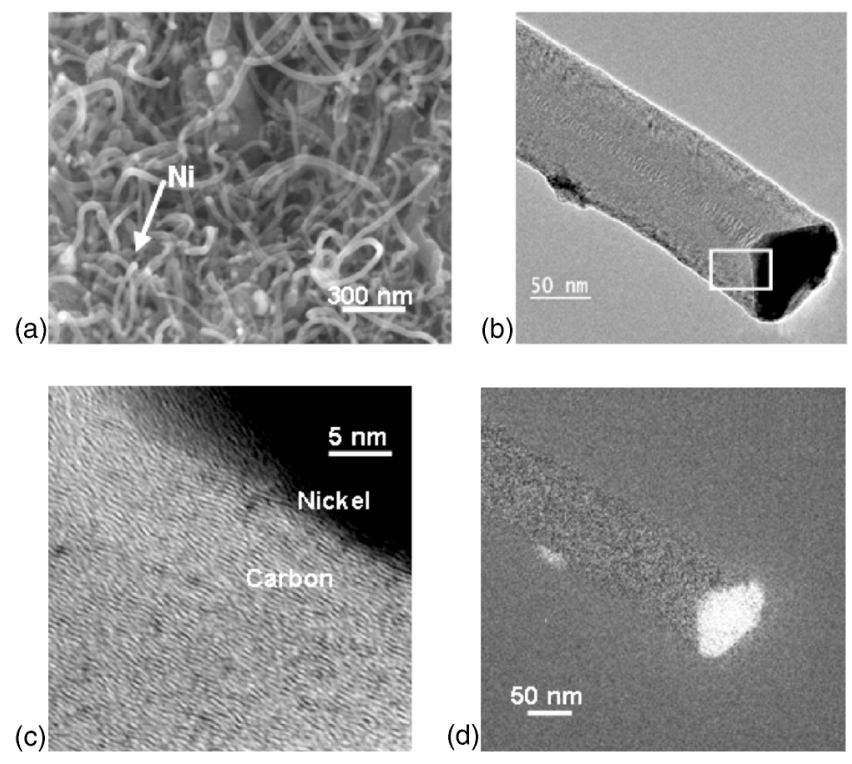

FIG. 2. Carbon nanofibers synthesized using rf supported MW PECVD at room temperature, at $100 \mathrm{~W}$ rf power, $250 \mathrm{~W}$ microwave power, and $70 \mathrm{sccm}$ methane flow rate: (a) SEM micrograph of room temperature deposited CNFs. (b) TEM micrograph of a CNF with a heart-shaped Ni catalyst particle at the tip, where the rectangle marks the area magnified in Fig. 2(c), (c) TEM image of the graphitic structure of the CNF, with planes parallel to the sides of the Ni catalyst, (d) energy filtered Ni chemical map of the CNF in Fig. 2(b), demonstrating that the particle at the tip is Ni, and that $\mathrm{Ni}$ does not diffuse into the CNF.

of the attached nickel particle, which usually is heart shaped and completely enveloped by a layer of carbon [Fig. 2(b) and 2(d)]. Bidirectional growth with a diamond-shaped catalyst particle in the middle of a CNF was also observed. Moreover, each nickel particle was responsible for the growth of single unidirectional or bidirectional CNF. Figure 2(c) reveals the presence of disordered graphene layers parallel to the surface of the heart-shaped catalyst particle, forming "herringbone" or "cup-stacked" structures with $\approx 0.34 \mathrm{~nm}$ as the interplanar distance. Figure 2(d) shows an energy-filtered TEM (EFTEM) image formed with electrons that have passed through the sample and have lost a unique amount of energy, which is characteristic to the Ni $L$ edge $(2 p-3 d$ transitions, $855 \mathrm{eV}$ ). Hence, Fig. 2(d) shows a Ni map, revealing that the particle at the tip of the $\mathrm{CNF}$ is indeed $\mathrm{Ni}$ and that $\mathrm{Ni}$ does not diffuse within the CNF to concentrations above the EFTEM detectability limit.

The TEM micrographs shown in Fig. 2 suggest that the tip growth mechanism, for carbon nanofibers grown in this study is similar to the tip growth processes described by Baker and Harris ${ }^{25,26}$ and used by Bower and co-workers ${ }^{27}$ to explain the growth of their CNTs using microwave PECVD. Prior to the growth of carbon nanofibers, formation of small domains of ultrafine transition metal particles occurs. These domains act as nucleation seeds for the carbon nanofiber growth. The size of small domains corresponds to the diameter of the CNF. After decomposition of methane on the surface of the ultrafine metal particles, carbon and hydrogen dissolves in the metal creating a supersaturated surface. Nickel metal particles become supersaturated by carbon, which then precipitates at the "cold-end" of the catalyst particle surface to form carbon nanofiber structures. All these 
methods require a high temperature to create the necessary conditions for the synthesis of CNFs. The mechanism involved in low temperature growth of CNF is not fully understood yet. We therefore believe the size of the nanodomains formed in the Ni catalyst is critical for the efficient coupling of the electromagnetic energy of excitation source to the particle. Although the macroscopic temperature of the substrates is low, enough localized energy is transferred into the catalyst particles to generate the nucleation sites and subsequent growth.

In addition to the well known unidirectional and bidirectional morphologies, between $10 \%$ and $20 \%$ of the CNFs illustrated in the present work are spontaneously branching or welding during growth, with the formation of $Y$-shaped junctions [Figs. 3(a)-3(c)]. The SEM micrograph in Figure 3(a) shows a number of welded CNFs, some of which are indicated by the arrows. Figures 3(b) and 3(c) show one such $Y$-type junction that has been tilted through $26^{\circ}$, showing that the CNFs are welded to form the connection. The axis of tilt is indicated in Fig. 3(c). Formation of carbon nanofiber junctions involves complex growth mechanism that is yet to be fully determined. As a possible growth mechanism to explain carbon nanotube and nanofiber junction formation a tip fusion was previously suggested. ${ }^{21,28}$ SEM and TEM investigations conducted did not provide evidence that the tip fusion had occurred at any stage of the growth process. The electric and magnetic field effects and localized energy transfer in the plasma could cause carbon-carbon bond breaking and redeposition of the carbon atom to form the junction. We believe that irradiation of the electrons and ions from the plasma play significant role in carbon nanotube and nanofiber welding and formation of the junctions. Terrones and co-workers demonstrated formation of molecular junctions of crossed single-wall CNTs (SWCNTs) using the electron beam in a transmission electron microscope at specimen temperatures of $800{ }^{\circ} \mathrm{C}^{29}$ Welding of crossed SWCNTs using ion beam irradiation was demonstrated employing empirical potential molecular dynamics. ${ }^{30}$ Authors have also suggested that ion beam irradiation potentially could be used for developing complicated networks of joined nanotubes. They concluded that welding of carbon nanotubes is not possible without structural defects. Defect rich structures of carbon nanofibers synthesized in this work, could be very beneficial for junction formation between them. Carbon nanotube and nanofiber junction formation in plasmas could be the proof for the role ion irradiation plays. More work is required to investigate plasma electron and ion characteristics during the synthesis of branched carbon nanofibers and establish the role of electron and ion irradiation.

Raman spectroscopy was used for structural analysis of carbon nanofibers synthesized using rf combined microwave PECVD. Raman spectra were taken under ambient conditions using a Renishaw RM 100 Raman Microscope (Fig. 4). The excitation source was a $514.5 \mathrm{~nm} \mathrm{Ar}^{+}$laser with optical power at the source of $20 \mathrm{~mW}$. The wave numbers of the main characteristic peaks in the spectra were determined by Lorentzian curve fitting. The Raman spectra of all forms of carbon show two common features: a $G$ peak at about $1580 \mathrm{~cm}^{-1}$ and a $D$ peak at about $1355 \mathrm{~cm}^{-1}$. The Raman
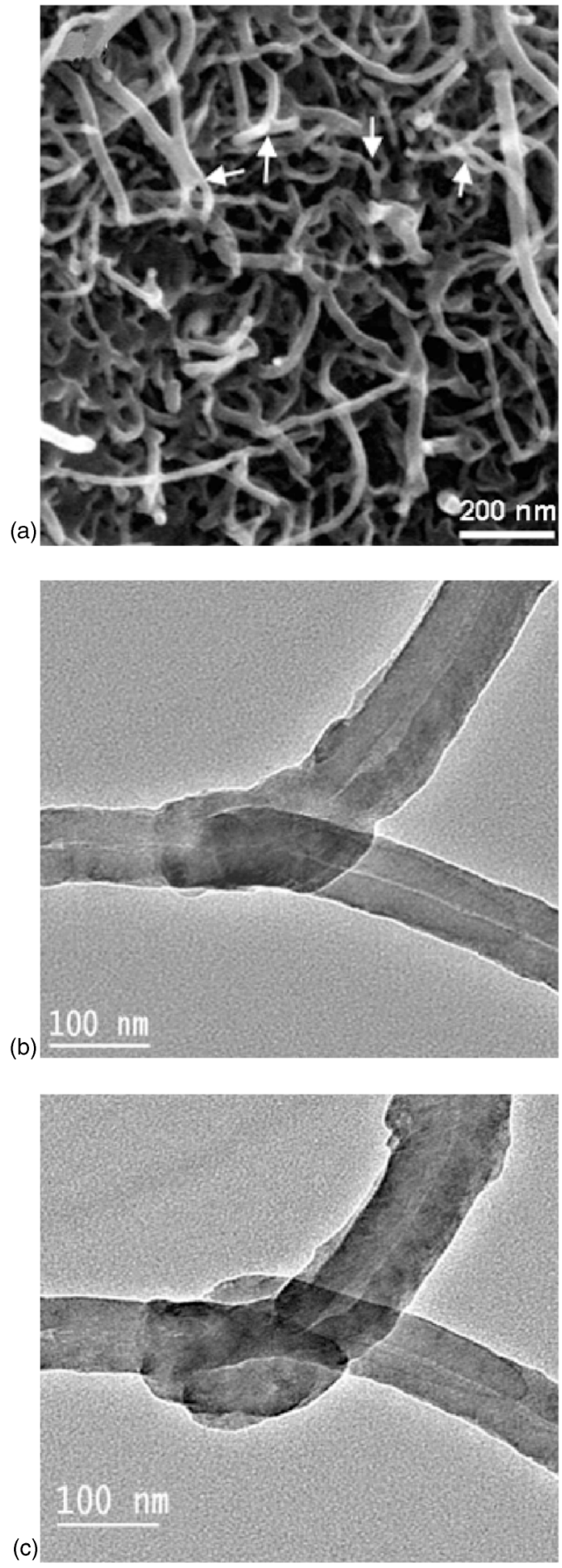

FIG. 3. Branched CNFs synthesized using rf supported MW PECVD at $100 \mathrm{~W}$ rf power, $850 \mathrm{~W}$ microwave power, and $70 \mathrm{sccm}$ methane flow rate at room temperature: (a) SEM micrograph showing area with branched $\mathrm{CNF}$ forming interconnecting network, (b) TEM image of $Y$-shaped CNF junction, (c) TEM image tilted for $26^{\circ}$ showing welded CNFs connection.

spectrum of carbon nanofibers synthesized at $500 \mathrm{~W}$ of microwave power shown in Fig. 4 has a $G$ peak position around $1577 \mathrm{~cm}^{-1}$, with a shoulder at around $1611 \mathrm{~cm}^{-1}$. The position of the defect induced $D$ peak is around $1346 \mathrm{~cm}^{-1}$, with a higher intensity than the $G$ peak $\left(I_{\mathrm{G}} / I_{\mathrm{D}}=0.85\right)$, indicating relatively small size graphite clusters within the CNF structure. The $G$ peak is due to the inplane bond stretching of all 


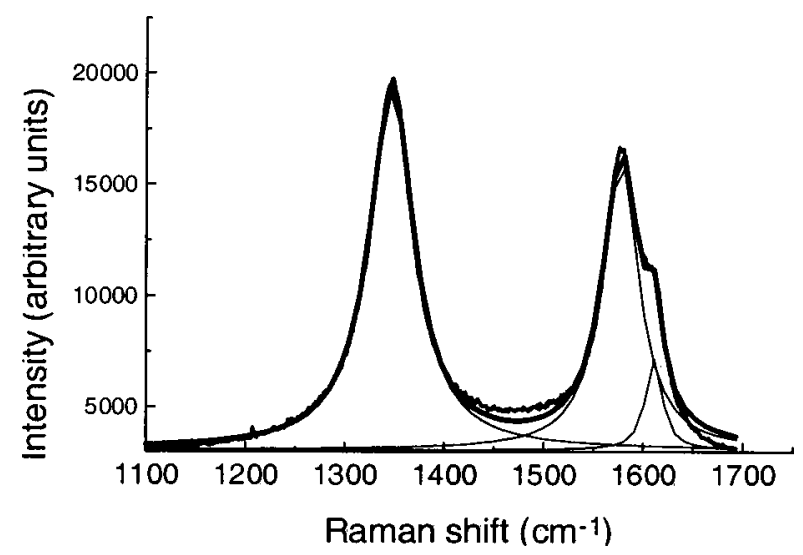

FIG. 4. Raman spectra of carbon nanofibers synthesized using rf supported MW PECVD $P_{\mathrm{RF}}=100 \mathrm{~W}, T=24{ }^{\circ} \mathrm{C}, \mathrm{CH}_{4}, f=70 \mathrm{sccm}$ at $P_{\mathrm{MW}}=500 \mathrm{~W}$, $\left(I_{\mathrm{G}} / I_{\mathrm{D}}=0.847\right)$.

pairs of $s p^{2}$ carbon atoms, and does not require the presence of sixfold carbon atom rings. ${ }^{31}$ The $D$ peak is forbidden in perfect graphite and only becomes active in the presence of disorder in graphene layer structures. Its intensity is connected to the presence of sixfold rings and represents the breathing modes of $s p^{2}$ atoms in the rings. ${ }^{31-33}$

\section{CONCLUSIONS}

Carbon nanofibers as well as branched nanofibers and their networks were produced at room temperature onto a variety of substrates, over relatively large areas. We have achieved the synthesis of CNFs at room temperature in a radio frequency assisted microwave PECVD system, using nickel powder as a catalyst at $100 \mathrm{~W}$ rf power and microwave power ranging from 250 to $850 \mathrm{~W}$. Room temperature growth of carbon nanofibers is of technological importance since it would allow the use of low temperature substrates which are suitable for flat panel display ${ }^{34}$ and fuel cell applications. ${ }^{35}$ Random networks of carbon nanotubes have already been used as electronic material for the fabrication into thin-film transistors. ${ }^{36}$ Electronic applications of carbon nanotube networks will benefit with the introduction of new temperature sensitive substrate materials when the method described in this manuscript is used for the carbon nanotube networks synthesis. Growth of CNTs and CNFs on temperature sensitive substrates could now be achieved using a method described in this paper, a rf PECVD method we reported earlier, ${ }^{22}$ and a dc PECVD method reported recently for the growth of vertically aligned carbon nanotubes at temperatures as low as $120^{\circ} \mathrm{C}$. ${ }^{23,27}$ However, only the technique described in this work demonstrates formation of $Y$-shaped CNF junctions and interconnecting networks at room temperature. This may be significant with respect to the possibility of creating interconnects for electronic devices at a nanometric scale and assembling new classes of materials from carbon nanofibers on temperature sensitive substrates, such as plastics, organics, and paper. The room-temperature carbon nanofiber growth will allow direct nanofiber network synthesis within temperature sensitive matrix materials for the improvement of the electrical and thermal conductivity, over relatively large areas, even at very low nanofiber concentrations.

\section{ACKNOWLEDGMENTS}

One of the authors B.O.B. would like to thank R. Devonshire and R. Jeans from University of Sheffield for use of the Raman spectrometer, and A. C. Ferrari from University of Cambridge for useful discussions.

${ }^{1}$ H. W. Kroto, J. R. Heath, S. C. O’Brien, R. F. Curl, and R. E. Smalley, Nature (London) 318, 162 (1985).

${ }^{2}$ S. Iijima, Nature (London) 354, 56 (1991).

${ }^{3}$ W. R. Davis, R. J. Slawson, and G. R. Rigby, Nature (London) 171, 756 (1953).

${ }^{4}$ N. M. Rodriguez, J. Mater. Res. 8, 3233 (1993).

${ }^{5}$ L. C. Qin, D. Zhou, A. R. Krauss, and D. M. Gruen, Appl. Phys. Lett. 72, 3437 (1998).

${ }^{6}$ S. H. Tsai, C. W. Chao, C. L. Lee, and H. C. Shih, Appl. Phys. Lett. 74, 3462 (1999).

${ }^{7}$ Y. C. Choi et al., Appl. Phys. Lett. 76, 2367 (2000).

${ }^{8}$ C. Bower, W. Zhu, S. Jin, and O. Zhou, Appl. Phys. Lett. 77, 830 (2000).

${ }^{9}$ M. Okai, T. Muneyoshi, T. Yaguchi, and S. Sasaki, Appl. Phys. Lett. 77, 3468 (2000).

${ }^{10}$ F. Hoshi et al., Diamond Relat. Mater. 10, 254 (2001).

${ }^{11}$ B. Q. Wei, R. Vajtai, and P. M. Ajayan, Appl. Phys. Lett. 79, 1172 (2001).

${ }^{12}$ M. J. Biercuk, M. C. Liaguno, M. Radisavljevic, J. K. Hyun, A. T. Johnson, and J. E. Fischer, Appl. Phys. Lett. 80, 2767 (2002).

${ }^{13}$ M. Menon and D. Sristava, Phys. Rev. Lett. 79, 4453 (1997).

${ }^{14}$ J. Li, C. Papadopoulos and J. M. Xu, Nature (London) 402, 253 (1999).

${ }^{15}$ P. L. McEuen, Nature (London) 393, 15 (1998).

${ }^{16}$ L. Kouwenhoven, Science 275, 1896 (1997).

${ }^{17}$ L. Chico, V. H. Crespi, L. X. Benedict, S. G. Louie, and M. L. Cohen, Phys. Rev. Lett. 76, 971 (1996).

${ }^{18}$ B. C. Satishkumar, P. J. Thomas, A. Govindaraj, and C. N. R. Rao, Appl. Phys. Lett. 77, 2350 (2000).

${ }^{19}$ W. Z. Li, J. G. Wen and Z. F. Ren, Appl. Phys. Lett. 79, 1879 (2001).

${ }^{20}$ J.-M. Ting and C.-C. Chang, Appl. Phys. Lett. 80, 324 (2002).

${ }^{21}$ G. W. Ho, A. T. S. Wee, and J. Lin, Appl. Phys. Lett. 79, 260 (2001).

${ }^{22}$ B. O. Boskovic, V. Stolojan, R. U. A. Khan, S. Haq, and S. R. P. Silva, Nat. Mater. 1, 165 (2002)

${ }^{23}$ S. Hofmann, C. Ducati, B. Kleinsorge, and J. Robertson, Appl. Phys. Lett. 83, 4661 (2003).

${ }^{24}$ A. Chambers, C. Park, R. T. K. Baker, and N. Rodriguez, J. Phys. Chem. B 102, 4253 (1998).

${ }^{25}$ R. T. K. Baker and P. S. Harris, in Chemistry and Physics of Carbon, edited by P. L. Walker, Jr. and P. A. Thrower (Marcel Dekker, New York, 1978), Vol. 14, p. 83.

${ }^{26}$ R. T. K. Baker, P. S. Harris, R. B. Thomas, and R. J. Waite, J. Catal. 30, 86 (1973).

${ }^{27}$ C. Bower, O. Zhou, W. Zhu, D. J. Werder, and S. Jin, Appl. Phys. Lett. 77, 2767 (2000).

${ }^{28}$ S. H. Tsai, C. T. Shiu, W. J. Jong, and H. C. Shih, Carbon 38, 1879 (2000).

${ }^{29}$ M. Terrones, F. Banhart, N. Grobert, J. C. Charlier, and H. Terrones, Phys. Rev. Lett. 89, 075505 (2002).

${ }^{30}$ A. V. Krasheninnikov, K. Nordlund, J. Keinonen, and F. Banhart, Phys. Rev. B 66, 245403 (2002);A. V. Krasheninnikov, K. Nordlund, J. Keinonen, and F. Banhart, Nucl. Instrum. Methods Phys. Res. B 202, 224 (2003).

${ }^{31}$ A. C. Ferrari and J. Robertson, Phys. Rev. B 61, 14095 (2000).

${ }^{32}$ C. Castiglioni, F. Negri, M. Rigolio, and G. Zerbi, J. Phys. Chem. 115, 3769 (2001).

${ }^{33}$ C. Thomsen and S. Reich, Phys. Rev. Lett. 85, 5214 (2000).

${ }^{34}$ F. Hoshi et al., Diamond Relat. Mater. 10, 254 (2001).

${ }^{35}$ A. Chambers, C. Park, R. T. K. Baker, and N. Rodriguez, J. Phys. Chem. B 102, 4253 (1998).

${ }^{36}$ E. S. Swon, J. P. Novak, P. M. Campbel, and D. Park, Appl. Phys. Lett. 82, 2145 (2003).

${ }^{37}$ S. Hofmann, C. Ducati, B. Kleinsorge, and J. Robertson, Appl. Phys. Lett. 83, 135 (2003). 